\title{
Correction: Surface- and tip-enhanced Raman scattering of bradykinin onto the colloidal suspended Ag surface
}

Cite this: Phys. Chem. Chem. Phys. 2015, 17, 19672

D. Swiech, ${ }^{\text {a }}$ Y. Ozaki, ${ }^{\text {b }}$ Y. Kim ${ }^{c}$ and E. Proniewicz ${ }^{{ }^{a}}$

DOI: $10.1039 / \mathrm{c} 5 \mathrm{cp} 90117 \mathrm{k}$

Correction for 'Surface- and tip-enhanced Raman scattering of bradykinin onto the colloidal suspended

www.rsc.org/pccp Ag surface' by D. Swiech et al., Phys. Chem. Chem. Phys., 2015, 17, 17140-17149.

The authors would like to add an additional Acknowledgment as shown below:

Kim gratefully acknowledges HUFS for financial support.

The Royal Society of Chemistry apologises for these errors and any consequent inconvenience to authors and readers.

\footnotetext{
${ }^{a}$ Faculty of Foundry Engineering, AGH University of Science and Technology, ul. Reymonta 23, 30-059 Kraków, Poland. E-mail: proniewi@agh.edu.pl

${ }^{b}$ Department of Chemistry, School of Science and Technology, Kwansei Gakuin University, Gakuen 2-1, Sanda, Hyogo 669-1337, Japan

${ }^{c}$ Department of Chemistry, Hankuk University of Foreign Studies, Yongin, Kyunggi-Do, 449-791, Korea
} 